\title{
Discovery of 15-second Oscillations in $H S T$ observations of W Sge following the 2001 Outburst
}

\author{
S. Araujo-Betancor, C. Knigge, T.R. Marsh R.I. Hynes ${ }^{1} \&$ D. Steeghs ${ }^{2}$ \\ University of Southampton, Dept. of Physics \& Astronomy, \\ Southampton SO17 1BJ, UK \\ K.S. Long \\ Space Telescope Science Institute, Baltimore, MD 21218, USA
}

\begin{abstract}
We report the discovery of $15 \mathrm{~s}$ oscillations in $H S T / \mathrm{STIS}$ far-UV spectroscopic observations of WZ Sge in decline, one month after the start of its 2001 outburst. We discuss the implications of this finding for both the magnetic and pulsating white dwarf models that have been proposed to account for the 28 s oscillations.
\end{abstract}

\section{Introduction}

WZ Sge is one of the most extreme CVs known: it has a very short orbital period $(82 \mathrm{~min})$, a very low mass ratio $(q=0.05)$, is one of the faintest $\mathrm{CVs}\left(M_{V} \simeq\right.$ 11.5) and has a 7-8 outburst amplitude recurring on a time scale of roughly $33 \mathrm{yr}$. Because of its extreme behaviour and characteristics, it is thought that WZ Sge is a highly evolved CV whose secondary is probably a brown dwarf-like object. WZ Sge went into outburst on 2001 July 23, around $10 \mathrm{yr}$ earlier than anticipated. We obtained HST/STIS far-UV spectroscopic observations to cover the immediate aftermath of the outburst. Fig. 1 (left) illustrates the timing of the three observing epochs, each consisting of $4 H S T$ orbits.

\section{Analysis of the oscillations}

Discrete Fourier transform inmediately revealed the presence of strong, rapid oscillations in the $H S T-3$ observations, though not in the data obtained in $H S T-1$ and HST-2. Fig. 1 (right) shows the discrete Fourier transform of the individual orbits in $H S T-3$. There are no significant peaks around the $28 \mathrm{~s}$ period seen in previous observations of the system during quiescence (e.g. Skidmore et al. 1999). The dominant frequencies near $15 \mathrm{~s}$ are clearly visible and in two of the orbits a signal at around $6.5 \mathrm{~s}$ can also be seen. The $15 \mathrm{~s}$ oscillations are quite strong (amplitude $\simeq 5 \%$ ) but not particularly coherent.

\footnotetext{
${ }^{1}$ Present address: The University of Texas at Austin, Austin, TX 78712, USA

${ }^{2}$ Present address: Harvard-Smithsonian Center for Astrophysics, Cambridge, MA 02138, USA
} 

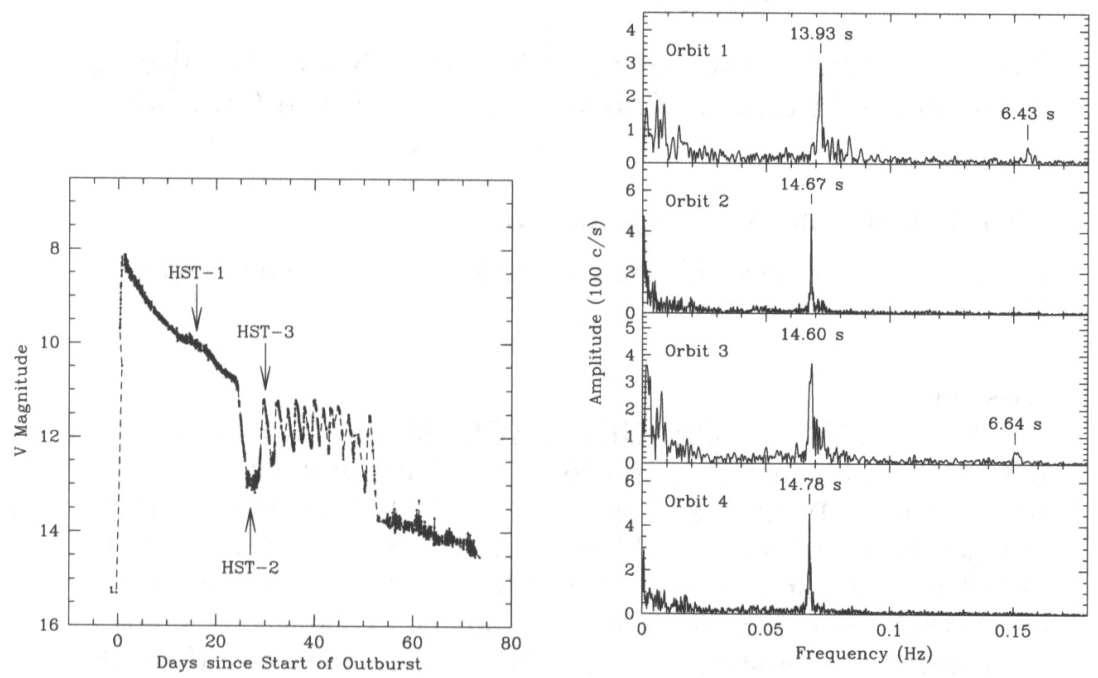

Figure 1. Right. Timing of the HST/STIS far-UV spectroscopic observations relative to the optical outburst light curve. Left. Discrete Fourier transform of the individual orbits in HST-3.

\section{Discussion}

It seems unlikely that the $15 \mathrm{~s}$ oscillations in our data can be explained by the ZZ Ceti-like white dwarf (WD) pulsations model favoured by Skidmore et al. (1999). Preliminary modelling of the FUV spectrum suggests $T_{\mathrm{WD}} \simeq 25,000 \mathrm{~K}$ during the time of our observations. This is well beyond the blue edge of the $\mathrm{ZZ}$ Ceti instability strip.

If we use the magnetic rotator model (see Patterson et al. 1998), the magnetosphere should have been completely crushed onto the WD surface as $\dot{M}$ in $H S T-3$ exceeded the quiescent rate by a factor in the range $150-15,000$.

An alternative magnetic rotator model proposed by Warner \& Woudt (2002) predicts a much weaker dependence of the magnetospheric radius on $\dot{M}$. Small $P_{\text {osc }}$ discontinuities occur as a magnetic reconnection events switch the feeding from one belt region to another. Long-term $P_{\text {osc }}$ changes reflect changes in $\dot{M}$. This model still needs to explain the $6.5 \mathrm{~s}$ oscillations as well as the absence/weakness of the oscillations in $H S T-1$ and $H S T-2$.

\section{References}

Patterson, J., Richman, H., Kemp, J., \& Mukai, K. 1998, PASP, 110, 403

Skidmore, W., Welsh, W. F., Wood, J. H., Catalán, M. S., \& Horne, K. 1999, MNRAS, 310,750

Warner, B., \& Woudt, P. A. 2002, MNRAS, in press (astro-ph/0204484) 